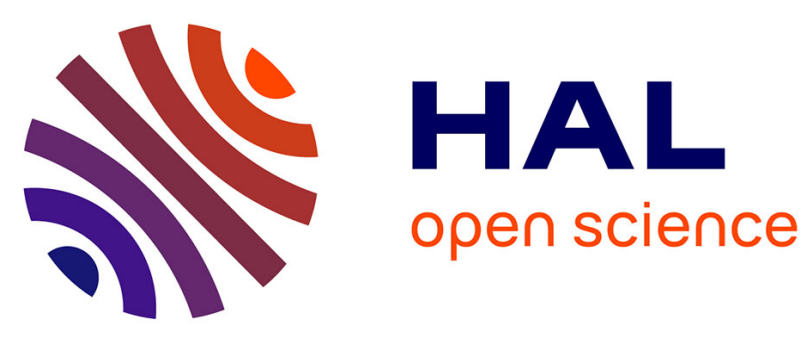

\title{
Un parcours de transmission
}

Catherine Cyssau

\section{To cite this version:}

Catherine Cyssau. Un parcours de transmission. Recherches en psychanalyse, 2005, 1 (3), pp.167 171. 10.3917/rep.003.0167 . hal-01502915

\section{HAL Id: hal-01502915 https://hal.science/hal-01502915}

Submitted on 6 Apr 2017

HAL is a multi-disciplinary open access archive for the deposit and dissemination of scientific research documents, whether they are published or not. The documents may come from teaching and research institutions in France or abroad, or from public or private research centers.
L'archive ouverte pluridisciplinaire HAL, est destinée au dépôt et à la diffusion de documents scientifiques de niveau recherche, publiés ou non, émanant des établissements d'enseignement et de recherche français ou étrangers, des laboratoires publics ou privés. 


\section{UN PARCOURS DE TRANSMISSION}

\section{Catherine Cyssau}

\section{Association Recherches en psychanalyse | « Recherches en psychanalyse »}

2005/1 n 3 | pages 167 à 171

ISSN 1767-5448

ISBN 2847950486

Article disponible en ligne à l'adresse :

http://www.cairn.info/revue-recherches-en-psychanalyse-2005-1-page-167.htm

\section{Pour citer cet article :}

Catherine Cyssau, «Un parcours de transmission », Recherches en psychanalyse 2005/1 (n ${ }^{\circ} 3$ ), p. 167-171.

DOI 10.3917/rep.003.0167

Distribution électronique Cairn.info pour Association Recherches en psychanalyse.

(C) Association Recherches en psychanalyse. Tous droits réservés pour tous pays.

La reproduction ou représentation de cet article, notamment par photocopie, n'est autorisée que dans les limites des conditions générales d'utilisation du site ou, le cas échéant, des conditions générales de la licence souscrite par votre établissement. Toute autre reproduction ou représentation, en tout ou partie, sous quelque forme et de quelque manière que ce soit, est interdite sauf accord préalable et écrit de l'éditeur, en dehors des cas prévus par la législation en vigueur en France. Il est précisé que son stockage dans une base de données est également interdit. 


\title{
Un parcours de transmission
}

\author{
Catherine Cyssau
}

Rendre hommage à Pierre Fédida fait être au cœur de l'humain, à ce point où il faut toucher à l'inhumain qui fait l'humain ${ }^{1}$. Inhumain est de ne plus parler avec lui, et de parler de lui, laissant dire les traces du silence, leurs legs de transmission que Pierre Fédida nous a laissés. L'extrême de l'inhumain peut, certes, conduire à la répression de cette affectation, au contact d'une dureté absolue de la mort parce qu'elle laisse l'individu démuni face à «l'impuissance psychique» ( Freud $\left.^{2}\right)$. Mais admettre l'inhumain tisse la communauté et détermine l'existence d'un parcours de transmission. La parole d'hommage est alors cette parole qui puise à la langue du «nous ». La perception d'une mémoire de la langue pourrait nous évoquer l'existence d'une source affective commune au langage du mythe et de la métaphore. A condition que ne soit pas méconnu l'écart d'un déplacement où le langage se sépare de la mémoire infantile qu'il retrouve. Pierre Fédida a été un penseur, il ne cherchait pas à insuffler sa pensée, il ne regroupait pas, il ne faisait pas école, il transmettait d'ailleurs moins un corpus théorique, qu'un mode aoristique de penser (Maldiney ${ }^{3}$ ), un moment poétique de construction des contraires ensemble ${ }^{4}$.

Comme interlocuteur, l'écriture de Pierre Fédida sous l'habitacle de sa voix nous a ouvert à la clairvoyance - ainsi qu'il l'écrivit - de «ce qu'on nomme entendre... entendre est pour l'homme s'entendre dans la mort ou encore... la mort est le seul véritable enjeu de l'entendu, tellement redouté que l'homme préfère comprendre » ${ }^{5}$. Sa pen-

1. Cela faisait partie des questions sur lesquelles Pierre Fédida a réfléchi, au cours des dernières années de son séminaire doctoral.

2. S. Freud (1912), Du rabaissement généralisé de la vie amoureuse, O.C. tome XI, p. 127-142.

3. H. Maldiney, Aîtres de la langue et demeures de la pensée, Lausanne, L'âge d'homme, 1975.

4. P. Fédida, Crise et contre-transfert, Paris, P.U.F., coll. Psychopathologie, 1992, p. 34-196.

5. Ibid., p. 43, 121. 
sée perlaborative semble presque faire partie du «nous», comme le travail du rêve demeure en négatif, invisible. On l'oublie, on en garde l'empreinte. Dans sa transmission, son mode de penser fonctionne, en même temps, comme la Chôra pour Platon ${ }^{6}$. Chôra sert de "porte-empreinte ». Mais elle ne garde, en elle-même, aucune trace des êtres qu'elle a conçus. La pensée de Pierre Fédida touche à l'horizon d'un point de perte $^{7}$. Elle n'a pas de remémoration. «Vertitur caelum, le ciel tourne » dit Virgile. Les latins avaient de l'horizon une vision verticale. Ils ne dissociaient pas les éléments, terre et ciel, du mouvement suivant lequel l'homme les habitait. Cela pourrait rendre compte du mode de transmission qui a marqué ceux que Fédida a formés.

La question est alors celle-ci. De quel point de vue accueillir la mémoire qui ne se souvient pas? Comment laisser se tresser le lieu d'écart d'un langage où insiste la résistance, sans céder à l'intention de l'acte ? Car l'intention de l'acte ne saurait alors éviter d'être meurtrière, elle n'y gagnerait pas d'être guerrière, mais se contenterait d'un détournement perversif. La sensibilité que dévoile Pierre Fédida au lieu, à la figure, à l'image, au matériau, l'entraîne là où le dessin dans la langue ouvre paradoxalement un saut du sens, s'il renonce à tout dessein. La transmission n'a pas de visée. Et la communauté psychanalytique de transmission peut ainsi rencontrer et provoquer le chemin de chacun.

L'écriture de Pierre Fédida échappe, en effet, au totalitarisme d'une menace de synthèse. Son approche singulière des processus critiques comporte un seuil d'éveil de l'imagination et du penser analytique. Le mouvement du texte sollicite, à la limite de ses nouages, cette mobilité psychique, perceptive d'une mémoire construisant avec l'infantile. L'écriture, de cette façon, engendre une transformation des images défascinées où se forme une théorie du symptôme. L'écriture, dans sa forme, son style, son temps et son contenu, garantit que le lecteur ne saurait venir occuper la place du destinataire de l'écrit. Elle retourne les concepts, comme s'élabore le visage inédit du nom qui s'entend ${ }^{8}$.

Entendre, dispose depuis l'angoisse d'une mémoire de l'oubli du rêve. Dans un texte de Fédida, daté de 1983, on peut lire: «La dignité tragique du langage connaît cet oubli vaillant par amour de ce qu'il sait. Ce qu'il sait n'est d'ailleurs pas vraiment un savoir. C'est plutôt un dire résonnant au cœur des hommes. Le veilleur de nuit, faute de sommeil, avait en lui la voix de son chant. Vaillant est donc l'oubli de son chant... Serait-ce la voix qui permet cet oubli du rêve de telle sorte que la parole soit gardienne de la liberté de tout dire et de l'interdit de dire tout... »9 .

L'interlocuteur serait, à l'écriture et à la pensée de Pierre Fédida, une puissance de nomination pour l'autre de l'absence. Cet invisible support transférentiel a cette fonction temporelle de rendre possible la transformation de l'étrangement infantile

6. P. Fédida, Théorie des lieux, Psychanalyse à l'Université, 1989, T. 14, n53, p. 3-16 \& n56, p. 3-18. \& J. Derrida., «Chôra », in Poikilia. Etudes offertes à J.-P. Vernant, Paris, éd. EHESS, 1987, p. 265-293.

7. C. Cyssau, Au lieu du geste, Paris, P.U.F., coll. Psychopathologie, p. 101 et suiv.

8. P. Fédida, Crise et contre transfert, op. cit., p. 66-272.

9. P. Fédida, L'Oubli du rêve, Furor 1983. 
en images, à partir du langage inhérent aux événements perceptifs des restes diurnes. Il est le mode d'engendrement des formes du fantasme. Pour que la perception donne lieu à langage et mémoire, l'analyste, dans la situation analytique, est placé entre la résonance $d u$ reste non-résolu - véritable point aveugle dont procède la fragmentation des images - et la reconnaissance de la fonction de reste diurne qu'il occupe. Le reste, littéralement, éveille «l'interlocuteur occulte» du symptôme ${ }^{10}$ si la perception, chez l'analyste, recueille le langage de ses figures et de cette façon, voit avec la mémoire de l'infantile.

Les morts sont encore ceux qui transforment le traumatique, et savent l'annoncer d'un rêve, en occupant la fonction de reste que prend l'événement de la vie. Leur image, par sa ressource affective, tisse ainsi l'ombre amnésique de la mémoire avec le cauchemar où leur figure éloigne l'insomnie, si celle-ci n'échappe pas au réveil. Alors, les morts sont d'étranges personnes dans nos rêves qui nous animent de leurs transferts... Car, dit Fédida, «La mémoire ne suffit pas à la substance des morts ». Leur matérialité d'âme, façonnée «des affects et des tonalités d'images » ${ }^{11}$, construit la mémoire de l'oubli et la réminiscence du rêve. Ce qui transforme le traumatique, c'est la fonction de reste que prend l'événement du jour. Le rêve en constitue la perception, à partir d'une virtualité métaphorique de langage dont doit disposer l'événement infinitésimal de la vie pour accéder à la fonction de reste diurne. Depuis ce matériau de l'image, la pulsion s'écrit dans la langue du mythe, et l'image, produisant l'écriture d'une théorie du symptôme - ce corps étranger intrapsychique, pour Freud ${ }^{12}$, accède à une mémoire de la langue.

Or poursuit Pierre Fédida en 1983: «L'acculturation contemporaine de la psychanalyse a produit une idéologie de l'insomniaque : tout ce qui se rapporte à la compréhension de soi et des autres, à la conscience de soi, à la connaissance du caché, voire même au renforcement du moi, nourrit les idéaux totalitaires de la sécurité des propriétaires méfiants... ${ }^{13}$

Il y dénonce dores et déjà, ainsi qu'il y reviendra ${ }^{14}$, un des risques d'abus ou de dérive de la supervision lorsqu'elle s'inscrit trop tôt précédant la construction d'une amnésie rêvante de l'analyse. Cette idéologie d'emprise insomniaque pourrait être consécutive à un mésusage de l'homosexualité dans la transmission. En effet, de l'hypnotiseur au complexe homosexuel du père chez le patient-homme associé au contre-transfert, ne faut-il pas convenir de l'obstacle auquel se heurte la question de la transmission, qui est celui de la croyance, voire du déni ? C'est la transmission homosexuelle du nom qui désigne le père du côté du langage. Mais ce disant, Pierre Fédida lie le processus de la croyance pour le rêve et le symptôme, avec une mimétique négative qui vaut pour l'analyste, l'apparence de l'image et la métaphore du lan-

10. Ibid., p. 244-275.

11. P. Fédida, Des bienfaits de la dépression, Paris, Odile Jacob, 2002.

12. P. Fédida, Crise et contre transfert, op. cit., p. 66-272.

13. P. Fédida, L'oubli du rêve, op. cit..

14. Cf. P. Fédida, Le site de l'étranger. La situation analytique, Paris, P.U.F., coll. Psychopathologie, 1993. 
gage. Si l'articulation du langage et de la mort relève d'abord d'une transmission, estil juste d'en limiter la portée poétique à la résistance du complexe homosexuel entre hommes ou à l'hallucination négative d'une croyance mimétique?

«L'oubli du rêve - retrouvé au fondement de la possible interprétation dès lors que celle-ci sera athée - inaugure le psychanalyste dans cette pré-figure du Veilleur de nuit qui n'est, après tout, qu'un reste diurne. C'est la seule marque qu'il porte de son aristocratie ${ }^{15}$.

Dans les rêves, voués à l'oubli, les morts peu nombreux qui ont compté n'ont pas d'adresse fixe, ils n'habitent nulle part, disparus, ils se cachent même impunément des vivants. Ils se manifestent à l'improviste par l'écriture qui nous lie à eux et qui les lie à nous. Adresse d'enveloppe dont l'expéditeur est désormais disparu, comme peut l'être l'effet étranger du texte que l'on a fini d'écrire et dont on est devenu, alors seulement peut-être, l'interlocuteur autre de soi-même. Ainsi les morts viennent à nous, dans leur disparition qui les incarnent et mobilisent la chance du rêve. Ils prennent des allers simples, l'image coupé de retour au familier, l'image si semblable touche au mort si elle gagne la parole qui désamorce la croyance en une mimesis de transmission. De même, il n'y a pas de supervision qui tienne sous les auspices de la séduction. Et c'est pourquoi, pensons-nous, le psychanalyste à l'université est le plus mal placé à l'égard d'une formation de supervision à la psychothérapie.

Est-ce ainsi que le rêve réveille l'apparence du dormant « qui touche au mort » ${ }^{16}$ ? Cette apparence du mort est, nous dit Pierre Fédida, l'interlocuteur $d u$ rêve ${ }^{17}$. Rêver n'est cependant pas voler. L'apparence immatérielle de l'image hallucinatoire du rêve interroge les limites du transfert métaphorique. La «désimagination de l'image » ${ }^{18}$, porterait finalement l'hystérie fantastique du corps, à cette bascule tout au cours de l'œuvre de Pierre Fédida, entre l'enjeu surréel d'un négatif de la perversion et l'expérience hyperréaliste d'une hypocondrie de la forme dans tous ses états ${ }^{19}$.

Catherine CYSSAU 6 impasse Marie Blanche 75018 Paris

15. P. Fédida, «L'Oubli du rêve», op. cit.

16. P. Fédida, Crise et contre-transfert, op. cit., p. 43-44.

17. Ibid., p. 219.

18. Ibid., p. 143.

19. Cf. P. Fédida, Le concept et la violence, Paris, 10/18, 1977 (regroupant des textes publiés entre 1967 et 1972); et «L'hypocondrie de l'expérience du corps », Psychopathologie de l'expérience du corps, Paris, Dunod, 2002, p. 107-159. 


\section{Catherine Cyssau - Un parcours de transmission}

Résumé: La question d'une communauté de transmission psychanalytique, dans ses liens avec l'écriture, le transfert, l'interlocuteur, le rêve, l'image, l'inhumain ainsi que le rôle de la mort et la fonction de l'oubli du rêve constituent des lignes d'approche de notre rencontre avec l'œuvre de P. Fédida.

Mots-clés : Transmission - Communauté - Ecriture - Interlocuteur - Rêve - Oubli Mort - Transfert - Image.

\section{Catherine Cyssau - A Transmission Process}

Summary : This article looks at the links between Pierre Fédida's work and the question of a community of psychoanalytic transmission, linked with writing, transference, the speaker, dreams, images and the inhuman, along with the role played by death and the function of forgetting dreams.

Key-words : Transmission - Community - Writing - Speaker - Dream - Forget Death - Transference - Image. 